\title{
Observation of Time-domain Rabi Oscillations in the Landau-Zener Regime with a Single Electronic Spin
}

\author{
Jingwei Zhou, ${ }^{1} \mathrm{Pu}$ Huang, ${ }^{1}$ Qi Zhang, ${ }^{1}$ Zixiang Wang, ${ }^{1}$ Tian Tan, ${ }^{1}$ \\ Xiangkun Xu, ${ }^{1}$ Fazhan Shi, ${ }^{1}$ Xing Rong, ${ }^{1}$ S. Ashhab,${ }^{2}$ and Jiangfeng $\mathrm{Du}{ }^{1,}, *$ \\ ${ }^{1}$ Hefei National Laboratory for Physics Sciences at Microscale and Department of Modern Physics, \\ University of Science and Technology of China, Hefei, 230026, China \\ ${ }^{2}$ Advanced Science Institute, RIKEN, Wako-shi, Saitama 351-0198, Japan
}

\begin{abstract}
Under resonant conditions, a long sequence of landau-zener transitions can lead to Rabi oscillations. Using a nitrogen-vacancy (NV) center spin in diamond, we investigated the interference between more than 100 Landau-Zener processes. We observed the new type of Rabi oscillations of the electron spin resulting from the interference between successive Landau-Zener processes in various regimes, including both slow and fast passages. The combination of the control techniques and the favorable coherent properties of NV centers provides an excellent experimental platform to study a variety of quantum dynamical phenomena.
\end{abstract}

PACS numbers: 03.67.Ac, 42.50.Dv

The phenomenon of Rabi oscillations, first studied in 1937 [1], occurs in almost any quantum system under the influence of resonant external driving and is at the heart of various spectroscopic techniques. Landau-Zener (LZ) transitions, first studied in 1932 [2 5], are intriguing phenomena that are ubiquitous in quantum systems, typically occurring when two energy levels of a quantum system undergo an avoided crossing.

Under suitable conditions LZ transitions can be treated as quantum coherent processes, and multiple such processes can interfere constructively or destructively [4]. Depending on the details of the interference, a long and regular sequence of LZ processes can exhibit resonance behavior similar to that seen in Rabi oscillations under weak driving conditions. The oscillations obtained in the case of constructive interference can therefore be seen as a manifestation of Rabi oscillations in the regime of ultrastrong driving [6]. Moreover, the interference between LZ processes gives rise to a number of novel features distinct from the case of weak driving. Particularly interesting is the fact that the patterns of the resonance lines vary drastically depending on whether each passage through the avoided crossing is slow or fast [6].

The experimental observation of Rabi oscillations in the LZ regime requires stringent conditions. The coherence time of the quantum system is required to be long enough to allow the coherent interference between multiple LZ processes, and the control fields are required to be accurate and stable in order to precisely adjust the quantum phases that govern the interference effects. In addition, the time resolution of the measurement needs to be high enough to allow the monitoring of the dynamics on short timescales.

With the recent advances in various quantum systems [7, 8, a number of experiments were able to demonstrate the controlled interference of LZ transitions. Interference between two LZ transitions has been observed in gaseous molecules [9], semiconductor-based quantum dots [10], NV centers [11], and atoms in optical lattices [12. Evidence for various interference effects involving multiple LZ transitions has been observed in the steady-state behavior of continuously driven superconducting qubits [13, 14 and NV centers [15. However, although those steady-state behaviors have been reported, the experimental observation of the abundant time-domain evolutions remains elusive due to more stringent conditions required.

Here by precise quantum control of the NV center in diamond at room temperature, we experimentally observed time-domain Rabi oscillations resulting from the interference between more than $100 \mathrm{LZ}$ processes. Our setup allows us to monitor the dynamics on both short and long timescales. On short timescales we clearly see the step-like dynamics that is characteristic of LZ transitions, while on long timescales we see oscillations that are characteristic of Rabi oscillations in a resonantly driven system. We further vary the parameters of our control fields in order to vary the degree of interference between successive LZ transitions. Our results therefore constitute a direct demonstration of fully controllable multi-transition interference in its most general form. All of our experimental results agree well with numerical simulations using parameters that are obtained from standard characterization techniques.

A NV center consists of a substitutional $\mathrm{N}$ atom with an adjacent vacant site $(\mathrm{V})$ in the diamond crystal lattice. It has an electron spin-1 ground state with three sublevels, $\left|m_{s}=0\right\rangle$ and $\left|m_{s}= \pm 1\right\rangle$, quantized along the [111] crystal axis [Fig. 1(a)]. Quantum logic gates [18, 19], quantum entanglement generation [20], single-shot readout 21 23 and quantum algorithms [24, 25] have been realized in such systems. These achievements make NV centers in diamond a promising candidate as a platform for building quantum computers. Furthermore, with their favorable coherence properties 
(a)

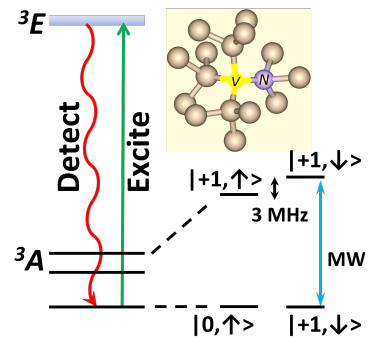

(b)

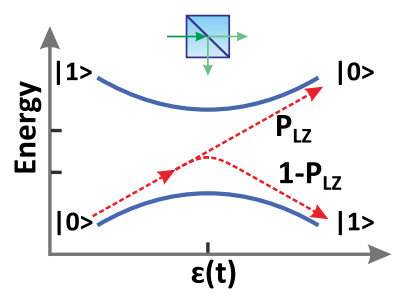

(d)
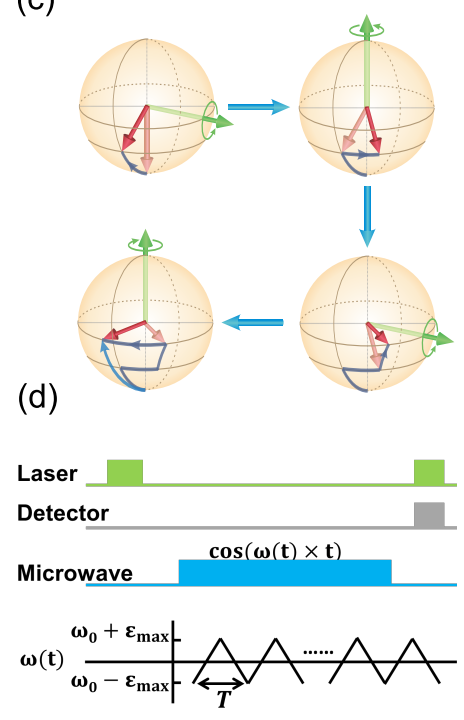

FIG. 1. (color online). (a) Schematic diagram of an NV center in the diamond lattice and the energy level diagram of the NV center. (b) A single Landau-Zener transition: when the energy levels undergo an avoided crossing, the quantum state undergoes a mixing process between the ground and excited states. This process is analogous to the operation of a beam splitter in optics experiments. (c)A single driving period contains four elementary rotations, which can be combined to produce a single effective rotation. On short timescales, one can see that each period involves four different rotations. On long timescales, the dynamics is described by the repeated application of the single-period rotation, as is the case for Rabi oscillations. (d) The pulse sequence used to drive Rabi oscillations in the LZ regime.

and atomic-scale size, NV centers have also been used for sensing magnetic field with nanometer spatial resolution [26, 27].

The sample we used is an isotopically pure ${ }^{12} \mathrm{C}$ (>99.9\%) diamond sample grown by chemical vapor deposition. When an externally applied magnetic field is aligned with the principal axis of the NV center, the Hamiltonian for the NV center is:

$$
H=D S_{z}^{2}+\gamma_{e} B S_{z}+A_{z z} I_{z} S_{z}
$$

Here $D=2.87 \mathrm{GHz}, \gamma_{e}=2.8 \mathrm{MHz} / \mathrm{G}, A_{z z}=-3.05 \mathrm{MHz}$ are parameters for the zero-field splitting, the Zeeman interaction, and the hyperfine interaction of the electron spin with the ${ }^{15} \mathrm{~N}$ nuclear spin $(I=1 / 2)$ of the NV center, respectively. In order to realize a simple two-level system, we apply a magnetic field of $\sim 510 \mathrm{G}$. This field lifts the degeneracy between the states $\left|m_{s}= \pm 1\right\rangle$. Furthermore, the nitrogen nuclear spins are polarized by optical pumping 28, such that $I_{z}=-1 / 2$ throughout the experiment. We use the states $\left|m_{s}=0\right\rangle$ and $\mid m_{s}=$ $+1\rangle$ for the qubit's states $|0\rangle$ and $|1\rangle$, respectively.

To induce transitions between the states $|0\rangle$ and $|1\rangle$, microwave (MW) fields are generated using a local (a)

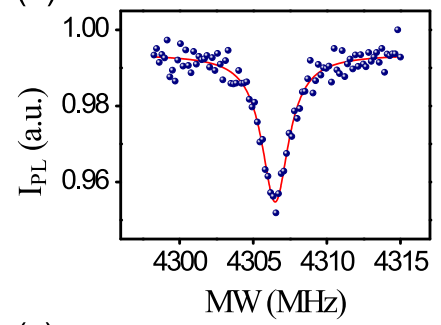

(c)

(b)

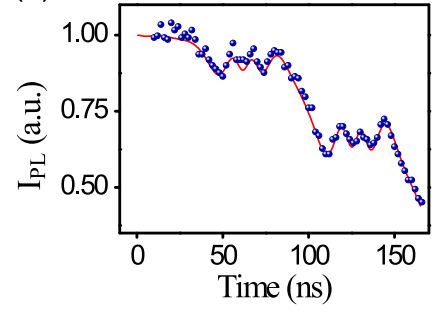

(d)
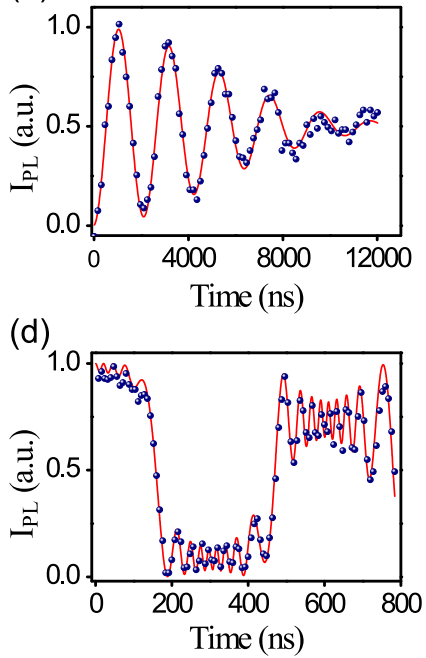

FIG. 2. (color online). (a) The optically detected magnetic resonance (ODMR) spectrum for the transition between the states $|0\rangle$ and $|1\rangle$ of the NV center (blue dots). The red curve is a fit with a single Lorentzian peak. (b) Results of a standard Ramsey experiment of the NV center. The results are fitted to the function $\exp \left[-\left(t / T_{2}^{*}\right)^{2}\right] \cos \left(2 \pi \delta_{f} t\right)$ $\left(\delta_{f}=0.56 \mathrm{MHz}\right)$, where the oscillations are due to the microwave detuning. (c,d) The occupation probability of the state $|0\rangle$ for a double-passage experiment in the fast- and slow-passage regimes.

oscillator mixed with an arbitrary-waveform generator and irradiated to the NV center via a coplanar waveguide. The form of the variable-frequency $\mathrm{MW}$ signal is $2 \Delta \cos [\omega(t) \cdot t]$. Since $\omega(t)$ remains close to $\omega_{0}$, we define the difference $\varepsilon(t)=\omega(t)-\omega_{0}$, i.e. $\varepsilon(t)$ is the offset between the microwave signal frequency and the resonance frequency of the $|0\rangle \leftrightarrow|1\rangle$ transition. If $\omega(t)$ is varied slowly compared to $\omega_{0}$ and $\Delta \ll \omega_{0}$, we can transform the Hamiltonian to a frame rotating with frequency $\omega_{0}$ and apply a rotating-wave approximation to simplify the Hamiltonian:

$$
H=\frac{\varepsilon(t)}{2} \sigma_{z}+\frac{\Delta}{2} \sigma_{x}
$$

Here we use a triangle wave for the function $\varepsilon(t)$ :

$$
\varepsilon(t)= \begin{cases}-\varepsilon_{\mathrm{m}}+\frac{4 \varepsilon_{\mathrm{m}}(t-n T)}{T}, & t \in\left(n, n+\frac{1}{2}\right) T \\ \varepsilon_{\mathrm{m}}-\frac{4 \varepsilon_{\mathrm{m}}(t-[n+1 / 2] T)}{T}, & t \in\left(n+\frac{1}{2}, n+1\right) T\end{cases}
$$

where $2 \varepsilon_{\mathrm{m}}$ and $T$ are, respectively, the range and period of the function $\varepsilon(t)$, and $n=0,1,2, \cdots$. When the system is initialized in the state $|0\rangle$ and $\varepsilon(t)$ is swept through the value zero, a simple LZ transition occurs. The probability of the transition from $|0\rangle$ to $|1\rangle$ is given by the LZ formula [Fig. 1(b)]

$$
P_{|0\rangle \rightarrow|1\rangle}=1-P_{\mathrm{LZ}}=1-\exp \left(-\frac{\pi}{2} \frac{\Delta^{2}}{v}\right)
$$


where $v$ is the sweep rate of the diabatic energy difference at the crossing. For the triangle wave used in our experiment the sweep rate is $4 \varepsilon_{\mathrm{m}} / T$.

Firstly, we characterize the parameters of the system. The optically detected magnetic resonance spectrum for the $|0\rangle \rightarrow|1\rangle$ transition is plotted in Fig. 2(a). The appearance of a single ESR resonance peak confirms the polarization of the nearby nuclear spin. The free-induction-decay signal, shown in Fig. 2(b), gives a rather long dephasing time of $T_{2}^{*}=6.56 \pm 0.17 \mu \mathrm{s}$ and spin-echo measurements (data not shown) give approximately $T_{2}=140 \mu \mathrm{s}$. For a slow sweep, the system transforms adiabatically from $|0\rangle$ to $|1\rangle$. As we make the sweep time shorter, $P_{\mathrm{LZ}}$ follows the exponential-decay function given in Eq. (3). Using this experiment we can extract the value of $\Delta$, and the values obtained this way always agree with the values obtained using resonant driving (see Supplemental Material). In Fig. 2(c,d), we show the results for double-passage LZS interferometry in the fast- and slow-passage regimes corresponding to $P_{\mathrm{LZ}}$ close to 1 and 0 , respectively. This short-time dynamics clearly shows the step-like LZ transitions and is in good agreement with the results of numerical simulations based on experimentally obtained parameters.

We now consider the effects of strong, periodic driving of the NV center. Landau-Zener-Stückelberg (LZS) interference can be understood using an analogy with Mach-Zehnder interferometry in an optical setup [13. This analogy provides a good understanding of the LZS setup when dealing with one or two LZ processes. For analyzing the resonance phenomena associated with continuous driving of the system, a geometric picture is more intuitive [14, 29]. When the two-level quantum system is driven through the avoided crossing region, the ground and excited states are mixed together in a process that can be described by the following matrix (See also Supplementary Material)

$$
N=\left(\begin{array}{cc}
\alpha & -\gamma^{*} \\
\gamma & \alpha^{*}
\end{array}\right)
$$

where $\alpha=\sqrt{1-P_{\mathrm{LZ}}} e^{-i \varphi}$ and $\gamma=\sqrt{P_{\mathrm{LZ}}}$, with $\varphi$ the geometric phase factor 6 .

In the geometric picture, the LZ mixing processes is represented by a rotation about an axis that depends on the various parameters described above. Between LZ processes the system is moved away from the avoided crossing and brought back some time later, such that a phase accumulates between the two quantum states. This phase is given by the integral over time of the energy separation between the two energy levels $2 \zeta=\int\left(E_{e}-\right.$ $\left.E_{g}\right) d t$, and the corresponding quantum process $U$ is represented by a rotation about the $z$ axis. A full driving cycle is therefore composed of four rotations alternating between two different axes [Fig. 1(c)]. This sequence of four rotations is equivalent to a single rotation $\left(G_{1}=\right.$
$\left.U_{2} N U_{1} N\right)$; depending on the various angles involved, the axis for the net rotation can point in any direction. Specifically, the matrix $G_{1}$ describing the net rotation has the same form as Eq. (4), but with parameters $\alpha$ and $\gamma$ that can be lengthy in general [6].

By stringing a large number of these rotations together, we obtain a resonance effect. If the axis of the single-period net rotation points in the $x y$ plane, the resonance condition is satisfied. Even if the mixing between the ground and the excited state in a single driving period is small, the rotations of multiple periods will add up constructively, and we obtain full oscillations between the states $|0\rangle$ and $|1\rangle$. If the axis of the single-period net rotation points outside the $x y$ plane, there can be partial, but not full, conversion between the two states. Two opposite limits are of particular interest: fast (almost sudden, $1-P_{\mathrm{LZ}} \ll 1$ ) and slow (almost adiabatic, $P_{\mathrm{LZ}} \ll 1$ ) passages. In both cases, we observe dynamics that reflects LZ processes associated with the passage through the avoided crossing as well as Rabi-oscillation dynamics on large timescales(Fig. 3).

For the data plotted in Fig. 3(a), we set $\Delta=5.57 \mathrm{MHz}$, $\varepsilon_{\mathrm{m}}=100 \mathrm{MHz}$ and $T=128 \mathrm{~ns}$, which gives $\varepsilon_{\mathrm{m}} / \Delta \approx 18$ (well in the $\mathrm{LZ}$ regime) and $P_{\mathrm{LZ}} \approx 0.91$ (fast-passage regime). We observe coherent oscillations that result from the quantum interference of a sequence containing over $100 \mathrm{LZ}$ processes. These oscillations are observed up to about $8 \mu$ s without any appreciable steady decay. For the data plotted in Fig. 3(b), we set $\Delta=9.60$ $\mathrm{MHz}, \varepsilon_{\mathrm{m}}=50.4 \mathrm{MHz}$ and $T=606 \mathrm{~ns}$, which gives $\varepsilon_{\mathrm{m}} / \Delta \approx 5$ (LZ regime) and $P_{\mathrm{LZ}} \approx 0.065$ (slow-passage regime). When we plot the data in the basis of the states $|0\rangle$ and $|1\rangle$, we can clearly see the steps associated with LZ processes. However, the long-time dynamics looks rather irregular and the oscillations are not easily visible. In order to show the oscillations more clearly, we transform the results to the adiabatic basis, as shown in Fig. 3(c). There we only keep data points for which $|\varepsilon(t)|>3 \Delta$, such that the difference between the diabatic and adiabatic bases is under $3 \%$ and the transformation of the state populations between the two bases can be done straightforwardly with negligible errors. The Rabi-oscillation pattern is now clearly recognizable. The observation of Rabi oscillations in this case is possibly more impressive than that in the fast-passage regime. Every passage through the avoided crossing is now almost adiabatic and drastically transforms the character of the quantum state. Nevertheless, quantum coherence is preserved and interference of over $15 \mathrm{LZ}$ processes is observed with no discernible decay. In Fig. 3(d) we show the data that correspond to the regime of intermediate sweep speed $\left(\Delta=5.84 \mathrm{MHz}, \varepsilon_{\mathrm{m}}=100 \mathrm{MHz}\right.$ and $T=592 \mathrm{~ns}$, which gives $\left.P_{\mathrm{LZ}}=0.61\right)$ and an intermediate degree of constructive interference between successive LZ processes. Specifically, the interference between two LZ processes within a single driving period is somewhat 
(a)

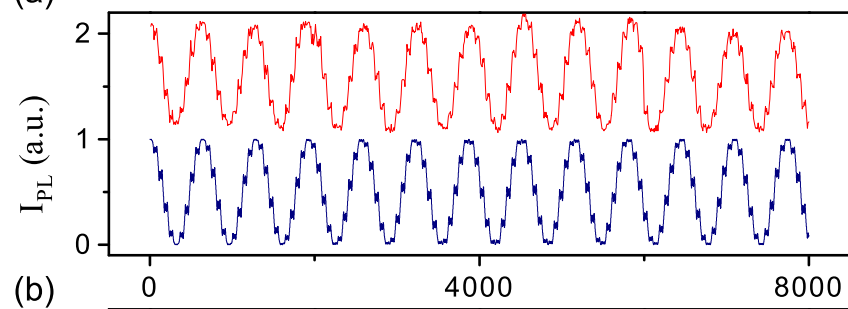

(b)

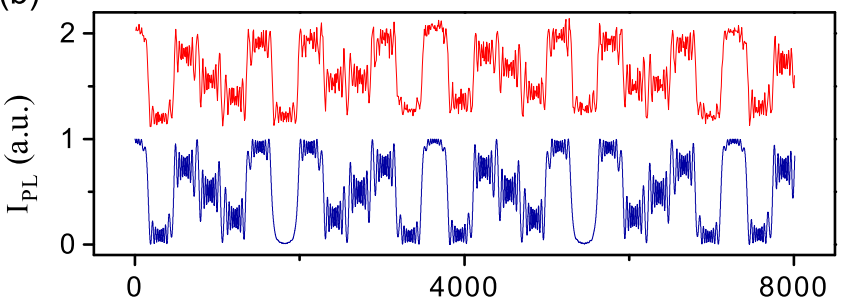

(c)

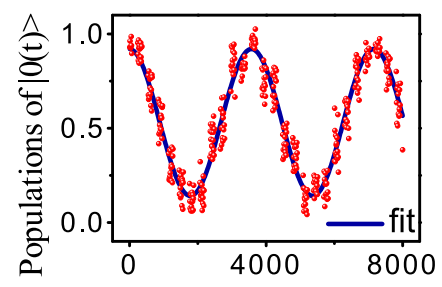

(d)

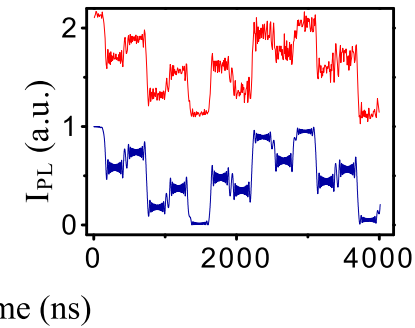

FIG. 3. (color online). Rabi oscillations resulting from the interference of many LZ processes. (a) fast-passage regime. The measured (pink line) and numerically simulated (blue line) occupation probabilities $P_{0}$ of the state as a function of time. The experimental data here and those in (b) and (d) are offset in y axis for visibility. The Rabi Frequency is about $1.49 \mathrm{MHz}$, which is consistent with numerical simulations. (b, c) slow-passage regime. Oscillations plotted the diabatic basis and in the adiabatic basis. (d) Dynamics in the regime of an intermediate sweep rate with partially destructive interference.

destructive, as can be seen from the fact that each step goes in the opposite direction from the previous one. The effect of varying the degree of constructive interference is also illustrated in Fig. 4. Taking the parameters of Fig. 3(a) and changing $T$ from $128 \mathrm{~ns}$ to $149 \mathrm{~ns}$, the constructive interference between successive LZ processes is replaced by completely destructive interference. This situation corresponds to the so-called coherent destruction of tunneling [30, where the $|0\rangle \rightarrow$ $|1\rangle$ transition is suppressed not because the driving is non-resonant, but because the parameters are chosen such that the single-period net rotation gives the identity operation (i.e. it has a zero rotation angle).

In summary, using a single electron spin in diamond, we have observed Rabi oscillations resulting from the quantum interference of more than $100 \mathrm{LZ}$ processes in various regimes ranging from slow to fast passage and from constructive to destructive interference within (a)
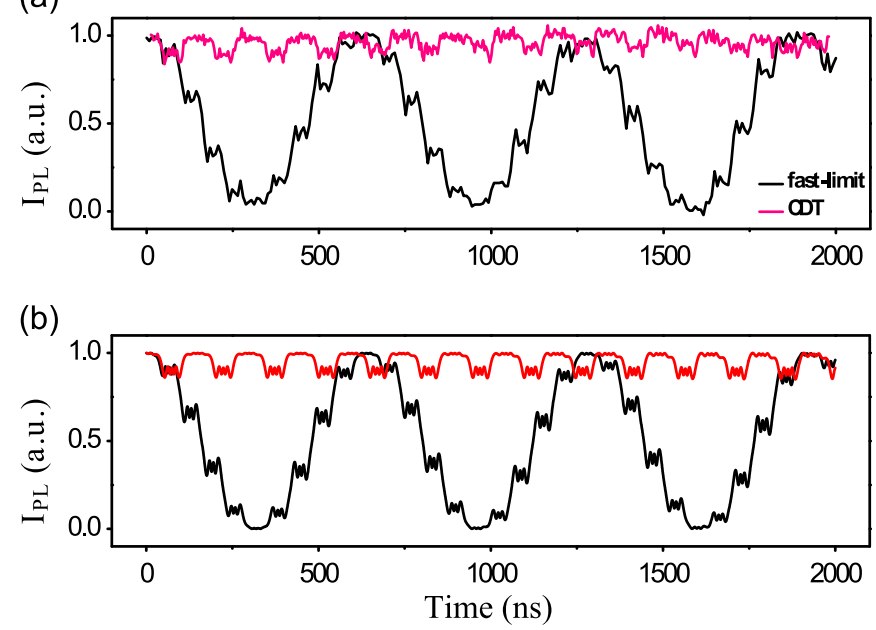

FIG. 4. (color online). Constructive versus destructive interferences in a single driving period. The parameters are the same as in Fig. 3(a), except for the sweep period being $T=149 \mathrm{~ns}$ in the case of destructive interference (pink line). The experimental results are shown in (a), while the results of numerical simulations are shown in (b). The case of destructive interference corresponds to the phenomenon of coherent destruction of tunneling.

a single driving period. The strong driving of the NV center has also allowed us to obtain the values of system parameters using alternative techniques, and the values extracted using multiple techniques have consistently agreed with one another. The experimental observation of the quantum oscillations in the LZ regime verifies the rich dynamics of a fundamental paradigm. The combination of precise control techniques with the favorable coherent properties of NV centers provide an excellent experimental platform with great potential in various fields, ranging from quantum information processing to the simulation of various dynamical phenomena in chemical and biology physics.

We thank J. Wrachtrup from University of Stuttgart, Germany for providing the isotopically purified diamond sample, Yiqun Wang in Suzhou Institute of Nano-Tech and Nano-Bionics for fabricating the coplanar waveguide, and Changkui Duan for helpful discussions. This work was supported by the National Key Basic Research Program of China (Grant No. 2013CB921800), the National Natural Science Foundation of China (Grant Nos. 11227901, 11275183, 91021005 and 10834005), the 'Strategic Priority Research Program (B)' of the CAS (Grant No. XDB01030400) and the Fundamental Research Funds for the Central Universities.

* djf@ustc.edu.cn 
[1] I. I. Rabi, Phys. Rev. 51, 652 (1937).

[2] L. Landau, Phys. Z. Sowjetunion 1, 88 (1932); ibid. 2, 46 (1932).

[3] C. Zener, Proc. R. Soc. Lond. A 137, 696 (1932).

[4] E. C. G. Stueckelberg, Helv. Phys. Acta 5, 369 (1932).

[5] E. Majorana, Nuovo Cimento 9, 43 (1932).

[6] S. N. Shevchenko, S. Ashhab, and F. Nori, Phys. Rep. 492, 1 (2010).

[7] T. D. Ladd, F. Jelezko, R. Laflamme, Y. Nakamura, C. Monroe, and J. L. O'Brien, Nature 464, 45 (2010).

[8] I. Buluta, S. Ashhab, and F. Nori, Rep. Prog. Phys. 74, 104401 (2011).

[9] M. Mark, T. Kraemer, P. Waldburger, J. Herbig, C. Chin, H.-C. Nägerl, and R. Grimm, Phys. Rev. Lett. 99, 113201 (2007).

[10] J. R. Petta, H. Lu, and A. C. Gossard, Science 327, 669 (2010).

[11] P. Huang, J. Zhou, F. Fang, X. Kong, X. Xu, C. Ju, and J. Du, Phys. Rev. X 1011003 (2011).

[12] S. Kling, T. Salger, C. Grossert, and M. Weitz, Phys. Rev. Lett. 105, 215301 (2010).

[13] W. D. Oliver, Y. Yu, J. C. Lee, K. K. Berggren, L. S. Levitov, and T. P. Orlando, Science 310, 1653 (2005).

[14] M. Sillanpää, T. Lehtinen, A. Paila, Yu. Makhlin, and P. Hakonen, Phys. Rev. Lett. 96, 187002 (2006).

[15] L. Childress and J. McIntyre, Phys. Rev. A 82, 033839 (2010).

[16] G. D. Fuchs, G. Burkard, P. V. Klimov, and D. D. Awschalom, Nature Phys. 7, 789 (2009).

[17] G. D. Fuchs, V. V. Dobrovitski, D. M. Toyli, F. J. Heremans, and D. D. Awschalom, Science 326, 1520 (2009).

[18] F. Jelezko, T. Gaebel, I. Popa, A. Gruber, and J. Wrachtrup, Phys. Rev. Lett. 92, 076401 (2004).

[19] F. Jelezko, T. Gaebel, I. Popa, M. Domhan, A. Gruber, and J. Wrachtrup, Phys. Rev. Lett. 93, 130501 (2004).

[20] P. Neumann, N. Mizuochi, F. Rempp, P. Hemmer, H. Watanabe, S. Yamasaki, V. Jacques, T. Gaebel, F. Jelezko, J. Wrachtrup, Science 320, 1326 (2008).

[21] B. B. Buckley, G. D. Fuchs, L. C. Bassett, D. D. Awschalom, Science 330, 1212 (2010).

[22] P. Neumann, J. Beck, M. Steiner, F. Rempp, H. Fedder, P. R. Hemmer, J. Wrachtrup and F. Jelezko, Science 329, 542 (2010).

[23] L. Robledo, L. Childress, H. Bernien, B. Hensen, Paul F. A. Alkemade, R. Hanson, Nature 477, 574 (2011).

[24] F. Shi, X. Rong, N. Xu, Y. Wang, J. Wu, B. Chong, X. Peng, J. Kniepert, R. Schoenfeld, W. Harneit, M. Feng, and J. Du, Phys. Rev. Lett. 105, 040504 (2010).

[25] T. van der Sar, Z. H. Wang, M. S. Blok, H. Bernien, T. H. Taminiau, D. M. Toyli, D. A. Lidar, D. D. Awschalom, R. Hanson and V. V. Dobrovitski, Nature 484, 82 (2012).

[26] G. Balasubramanian, I. Y. Chan, R. Kolesov, M. AlHmoud, J. Tisler, C. Shin, C. Kim, A. Wojcik, Philip R. Hemmer, A. Krueger, T. Hanke, A. Leitenstorfer, R. Bratschitsch, F. Jelezko and J. Wrachtrup, Nature 455, 648 (2011).

[27] J. R. Maze, P. L. Stanwix, J. S. Hodges, S. Hong, J. M. Taylor, P. Cappellaro, L. Jiang, M. V. Gurudev Dutt, E. Togan, A. S. Zibrov, A. Yacoby, R. L. Walsworth and M. D. Lukin, Nature 455, 644 (2011).

[28] V. Jacques, P. Neumann, J. Beck, M. Markham, D. Twitchen, J. Meijer, F. Kaiser, G. Balasubramanian, F. Jelezko, and J. Wrachtrup, Phys. Rev. Lett. 102, 057403
(2009).

[29] S. Ashhab, J. R. Johansson, A. M. Zagoskin, and F. Nori, Phys. Rev. A 75, 063414 (2007).

[30] F. Grossmann, T. Dittrich, P. Jung, and P. Hänggi, Phys. Rev. Lett. 67, 516 (1991). 